\title{
CARTOGRAFIA DE USO DO SOLO EM AMBIENTE URBANO ANÁLISE ORIENTADA POR OBJECTO DE IMAGENS QUICKBIRD*
}

\author{
SARA ENCARNAÇÃO ${ }^{1}$ \\ JORGE ROCHA ${ }^{2}$ \\ José ANTÓNIO TENEDÓRIO ${ }^{1}$ \\ ROSSANA ESTANQUEIRO ${ }^{1}$
}

\begin{abstract}
Resumo - Neste artigo é apresentada uma metodologia de análise de imagem orientada por objecto para separar duas classes de uso do solo: habitação unifamiliar e plurifamiliar. Estas classes, frequentes em áreas periurbanas, são muito heterogéneas do ponto de vista espectral, razão pela qual a abordagem pixel a pixel não serve para a sua correcta identificação. A análise orientada por objecto mostrou-se adequada para separar estas classes por se basear na segmentação multiresolução e nos parâmetros escala, cor, forma, suavidade e compacticidade. Os conceitos de habitação unifamiliar e de habitação plurifamiliar são discutidos tendo em consideração uma noção nova: a noção de objecto compósito.
\end{abstract}

Palavras-chave: Detecção remota, segmentação multiresolução, classificação hierárquica, objecto.

\begin{abstract}
LAND USE MAPPING OF URBAN AREAS USING OBJECT-ORIENTED ANALYSIS OF QUICKBIRD IMAGES. This article introduces a new object-oriented image analysis methodology, whose aim is to distinguish between two types of land use: single-family and multi-family households. These categories, which are especially common in peri-urban areas, are so heterogeneous from the spectral point of view that a per-pixel approach does not allow for their unambiguous identification. Object-oriented analysis has proven suitable to distinguishing between these two categories, insofar as it is based on multi-resolution segmentation and on the scale, color, form, smoothness and compactness parameters. The concepts of single-family and multi-family households are discussed by means of reference to a new notion - that of composite object.
\end{abstract}

Key words: Remote sensing, multiresolution segmentation, hierarchical classification, object.

* Recebido: 9/02/2006. Revisto: 1/06/2006; 4/04/2007; 18/10/2007. Aceite: 18/11/2007.

1 e-GEO, Centro de Estudos de Geografia e Planeamento Regional, Faculdade de Ciências Sociais e Humanas - Universidade Nova de Lisboa. E-mail: sara.encarnacao@fcsh.unl.pt, ja.tenedorio@fcsh.unl.pt, e rossana.estanqueiro@fcsh.unl.pt

2 Centro de Estudos Geográficos, Universidade de Lisboa. E-mail: jrocha@fl.ul.pt 
Résumé - CARTOGRAPHIE DE L'Utilisation DES SOlS EN MILIEU URBAin. UNE ANALYSE PAR OBJETS D'IMAGES QUICKBIRD. On présente une méthode d'analyse des images par objets, destinée à séparer deux classes d'utilisation du sol : les habitations unifamiliales et plurifamiliales. Ces classes, fréquentes en milieu périurbain, sont très hétérogènes du point de vue spectral, ce qui rendrait confuse une analyse pixel à pixel. L'analyse par objet s'est montrée efficace pour la séparation de ces deux classes, parce qu'elle est basée sur une segmentation multirésolution et sur les paramètres échelle, couleur, forme, continuité et compacité. Les notions d'habitation unifamiliale et plurifamiliale sont aussi discutées en fonction d'une autre notion, celle d'objet composite.

Mots-clés: Télédétection, segmentation multirésolution, classification híerarchique, objet.

\section{INTRODUÇÃO}

As dinâmicas envolvidas nos processos espaciais, resultado da integração de factores físicos, demográficos, económicos e políticos, entre outros, podem apresentar como suporte de interpretação e formalização as cartas de uso do solo. A informação espacial é estruturada em classes de uso do solo resultantes da interpretação de áreas "homogéneas", levando à identificação de formas e processos espaciais.

Em Detecção Remota, as formas e as estruturas espaciais encontradas nas imagens são dependentes de factores como o tipo de dados, as técnicas utilizadas e a informação a extrair. O pormenor a incorporar e o grau de precisão dos resultados são dependentes dos objectivos definidos à partida, estando, nesta fase, subjacente o conceito de generalização semântica e gráfica dos dados a tratar. Em meio urbano, por exemplo, a heterogeneidade do espaço exige um complexo sistema de definição de metodologias e dados a utilizar. A variabilidade das escalas de representação é, muitas vezes e simultaneamente, uma causa e consequência dos métodos e das técnicas a desenvolver.

$\mathrm{O}$ aumento da resolução espacial dos satélites e o pormenor contido na imagem tem trazido novos contornos à identificação e interpretação do uso do solo, a grandes escalas, o que origina novas desagregações das nomenclaturas a utilizar. Contudo, as classificações automáticas ou semiautomáticas ainda não apresentam a precisão desejada, principalmente em áreas fortemente heterogéneas, como são as áreas urbanas. Esta dificuldade prende-se não só com a complexidade geométrica/espacial destes territórios mas também com a complexidade semântica das suas entidades físicas, isto é, a identificação de algumas classes de uso não depende tanto das características físicas dos seus objectos, mas das funções a estes atribuídas. As abordagens usuais ao nível do pixel apresentam uma dificuldade própria, na medida em que trabalham sobretudo com a dimensão da resposta espectral dos objectos presentes na imagem, quando a diferenciação empírica desses mesmos objectos incorpora outras dimensões inerentes à natureza física e social do objecto. 


\section{ENQUADRAMENTO TEÓRICO}

\section{Abordagem orientada por objecto}

$\mathrm{Na}$ abordagem orientada por objecto a unidade mínima de análise é o objecto e é sobre ele que decorrem as operações de classificação baseadas em regras de conhecimento, construídas a partir dos diversos atributos desses objectos, como sejam a cor, a forma, a hierarquia, a textura e o contexto espacial. Esta abordagem procura classificar a informação presente na imagem, a partir da formalização das relações estabelecidas entre as entidades encontradas, entendendo-se a imagem como um espaço próprio de construção e representação da realidade. A classificação é assim entendida como um resultado da delimitação de fronteiras, não apenas de "corpos" singulares e homogéneos, mas acima de tudo como um entendimento de novas concepções de espaço com diferentes propriedades e objectos.

Neste sentido, parte-se da hipótese de que a diferenciação de um objecto singular (elemento construtor) em relação a outros, espacialmente relacionados, passará pela introdução de atributos de aproximação ao objecto real, como são disso exemplo a textura, a localização relativa e o contexto espacial. Assim, a criação de redes de objectos dá origem a uma tipologia de classes hierarquizada, a várias escalas de percepção do objecto, numa abordagem sistémica, representativa do uso e ocupação do território.

\section{A noção de objecto em classificação de imagem}

J. Nunes (1991), citando Chapman $(1975,1978)$, apresenta a conceptualização da noção de espaço geográfico como:

1) Um conjunto de objectos com propriedades espaciais (espaço relativo);

2) Um conjunto de localizações com propriedades temáticas (espaço absoluto).

Os eventos apreendidos pelo utilizador dependem do tipo de dados, do conhecimento de campo, do conhecimento do significado de uma imagem no que respeita à tradução dos valores numéricos em cor e depois em significado, do software utilizado, da formação do utilizador e do objectivo de análise. A taxonomia das construções formadas são também dependentes destes factores.

$\mathrm{Na}$ imagem, os objectos criados devem ajustar-se aos objectos da realidade, tanto ao nível da sua geometria, i.e., os seus limites/forma, como ao nível da sua semântica, i.e., conteúdo/significado. Qual a natureza do objecto? Que significado é atribuído a cada objecto de acordo com o que ele representa? Destas interrogações surge a noção de objecto compósito: 
1) Se entendido como um todo, representa o objecto geográfico e, acima de tudo, a funcionalidade desse mesmo objecto. Neste sentido, aproximase do objecto construído pela cognição humana;

2) Se entendido como um todo composto por partes que o definem e caracterizam. A interligação das diferentes partes produz um objecto compósito.

A percepção situar-se-á, desta forma, num campo inicial de análise intuitiva da imagem de satélite, em que o intérprete identifica formas, texturas, cores, padrões de organização, formulando a estrutura do método a utilizar para interpretação da imagem. As tomadas de decisão no domínio pragmático, tomam usualmente a ordem inversa, no que diz respeito à definição dos objectos para a determinação das suas funções. A função é a simplificação semântica do objecto entendido como um todo, determinada pelas partes constituintes desse mesmo objecto, que ao nível conceptual, apresentam possibilidades infinitas de opções combinatórias de desagregação. Desta forma, revela-se necessário partir da simplificação semântica e gráfica, isto é, da determinação das funções a encontrar, para a determinação da complexidade desta mesma generalização semântica e gráfica, procurando e formalizando as componentes que dela fazem parte.

Defende-se, então, a utilização do objecto como unidade mínima de análise, na medida em que do uso do pixel decorre uma perda de significado entre o objecto representado e aquele que o representa, na medida em que o pixel é uma unidade discreta e independente, para a qual as relações de vizinhança são dadas apenas por um tratamento estatístico do seu valor digital.

\section{CONSTRUÇÃO DE OBJECTOS}

A construção de objectos por segmentação multiresolução (Baatz e Schäpe, 2000), baseia-se na partição da imagem em segmentos a diferentes escalas de percepção. As imagens criadas são objectos construídos a partir da forma e significado. A forma do objecto resulta de um conjunto complexo de regras visuais inerentes ao processo cognitivo que permite segmentar o espaço visual em subunidades com determinado significado. $\mathrm{O}$ indivíduo tem a capacidade de trabalhar numa interacção de escalas, com elementos discretos e contínuos, numa perspectiva relativista, em relação ao objecto de foco, isto é, o indivíduo tem a capacidade de interpretar, simultaneamente, um objecto de interesse e a sua envolvente, estabelecendo, dessa forma, um entendimento sobre o território ou superfície de observação. Com a abordagem orientada por objecto procura-se uma aproximação a esta forma de construção de objectos, a partir de uma imagem. Segundo Haralick e Shapiro (1985), os objectos construídos com a segmentação de imagem devem ser "uniformes e homogéneos relativamente a algumas características como o tom de cinzento ou a textura. $\mathrm{O}$ interior das regiões 
deve ser simples e desprovido de muitos e pequenos buracos (...). O limite de cada segmento deve ser simples e espacialmente exacto".

Em eCognition (Baatz e Schäpe, 2000) os objectos são construídos por segmentação multiresolução, uma técnica de crescimento de regiões. Neste processo, os objectos serão fundidos ou não, de acordo com critérios de decisão, nomeadamente o critério de homogeneidade $(h)$ e o parâmetro de escala (Baatz et al., 2003). Na definição do grau de homogeneidade, a vizinhança entre objectos é calculada segundo um determinado espaço característico (feature space), dado por exemplo pelos valores espectrais. Desta forma, o grau de ajustamento (degree of fitting) $(h)$ é determinado, segundo $n$-dimensionalidades $(d)$ de espaços característicos (feature spaces) (f), por:

$$
h=\sqrt{\sum_{d}\left(f_{1 d}-f_{2 d}\right)^{2}}
$$

Posteriormente, as distâncias podem ser normalizadas através da equação (2):

$$
h=\sqrt{\sum_{d}\left(\frac{f_{1 d}-f_{2 d}}{\sigma_{\beta}}\right)^{2}}
$$

O processo procura a minimização da heterogeneidade, tendo em conta a dimensão do objecto determinada pelo parâmetro de escala. De forma a optimizar a delimitação do objecto, em relação às suas propriedades espectrais e espaciais, o critério geral para a fusão de objectos (3) integra os critérios de heterogeneidade espectral $\left(h_{c o r}\right)(4)$ e heterogeneidade espacial $\left(h_{\text {forma }}\right)(5)$, sendo definido por:

$$
f=w+h_{c o r}+(1-w)+h_{\text {forma }}
$$

Sendo $w$ o peso do parâmetro cor $\left(h_{c o r}\right)$, variando este entre 0 e $1 ; n$ a dimensão do objecto e $\mathrm{w}_{\mathrm{c}} \mathrm{o}$ peso de cada canal $c$.

$$
h_{c o r}=\sum_{c} w_{c}\left(n_{\text {merge }} * \sigma_{c}^{\text {merge }}-\left(n_{\text {obj } 1} * \sigma_{c}^{\text {obj } 1}+n_{o b j 2} * \sigma_{c}^{o b j 2}\right)\right.
$$

O critério de forma $\left(h_{\text {forma }}\right)$, que varia entre 0 e 1 , é construído por dois critérios de optimização da forma do objecto, isto é, a compacticidade $\left(h_{\text {cmpct }}\right)$ (6) e a suavidade $\left(h_{\text {suav }}\right)(7)$, variando também estes entre 0 e 1 :

$$
h_{\text {forma }}=w_{\text {cmpct }} * h_{\text {cmpct }}+\left(1-w_{\text {cmpct }}\right)^{*} h_{\text {suav }}
$$




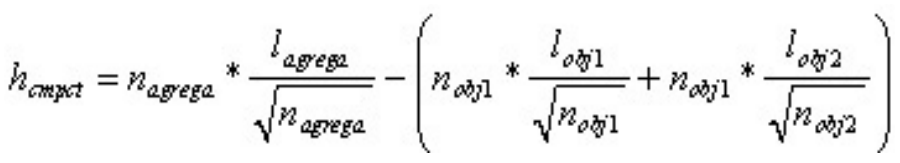

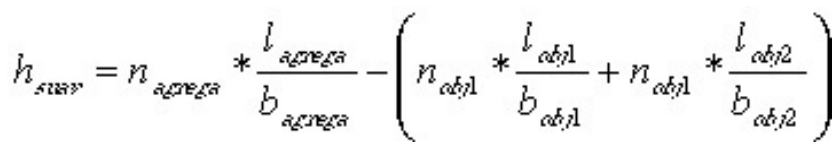

Sendo $n$ a dimensão do objecto em pixels, $l$ o perímetro do objecto e $b$ o perímetro da caixa de delimitação.

O máximo de heterogeneidade permitida na construção do objecto - isto é, a dimensão do mesmo, é determinado pelo parâmetro de escala. No processo de fusão de objectos é calculada a diferença entre a raiz quadrada do parâmetro de escala e o valor geral de fusão $f$. Se o valor $f$ for superior ao admitido pelo parâmetro de escala, o objecto não será fundido. Desta forma, quanto menor o parâmetro de escala maior será o número de objectos a construir, já que o grau de liberdade para aumentar a heterogeneidade do objecto é reduzido, resultando num objecto de pequena dimensão. Pelo contrário, se o parâmetro de escala for superior, o número total de objectos construídos será menor, embora de maior dimensão. Estes níveis produzem uma rede hierárquica de objectos, a partir da qual um determinado objecto conhece os seus vizinhos, os seus sub e superobjectos (Baatz et al., 2003), permitindo futuras regras de classificação baseadas em hierarquia de relações entre objectos.

A escolha do conjunto de parâmetros a utilizar é função dos objectivos e das características das imagens, nomeadamente da variância, da resolução e do tamanho das mesmas.

$\mathrm{Na}$ figura que se segue apresentam-se alguns exemplos de experimentação de segmentação multiresolução, para uma mesma imagem, mantendo o parâmetro de escala a 25 e fazendo variar os parâmetros de cor e forma.

Das figuras constata-se, como seria de esperar, que nos casos em que existe um maior peso atribuído à cor, resultam objectos que se assemelham aos encontrados por análise visual, na medida em que, ao nível do pixel, a cor apresenta maior contraste do que a forma, ou seja, a heterogeneidade entre objectos é conseguida através dos seus valores digitais.

Desta forma, é necessário definir à partida quais os objectos de interesse a procurar na imagem, de acordo com o objectivo pretendido. No presente caso, a definição dos objectos de interesse deve obrigatoriamente englobar os seguintes aspectos, de acordo com a sua função no processo de classificação:

a) Os objectos a serem classificados devem adequar-se à semântica da hierarquia da classificação, nomeadamente a passagem de classes de ocupação do solo para classes de uso do solo. 


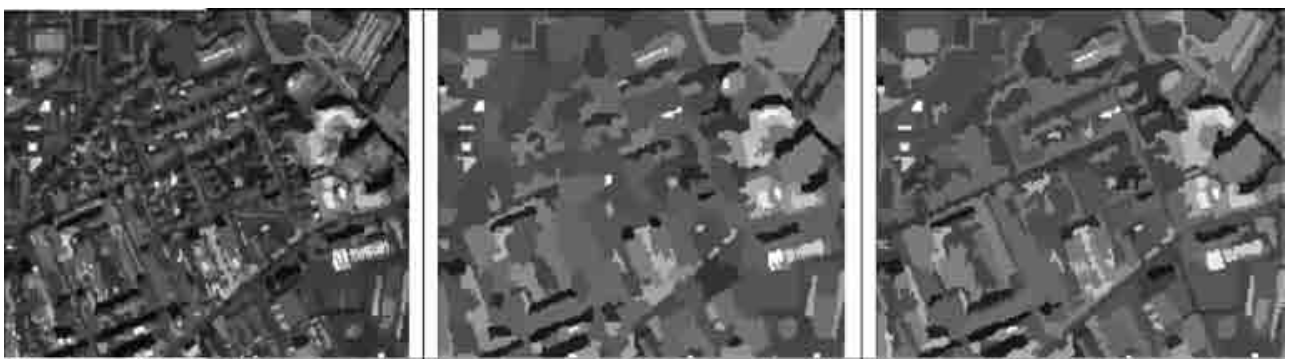

Fig. 1 - Exemplos de segmentações com variação dos pesos de cor e forma:

a) Cor: 1 ; forma: 0 ,

b) Cor: 0,2; forma: 0,8; compacticidade: 0,9; suavidade: 0,1,

c) Cor: 0,2; forma: 0,8; compacticidade: 0,1 ; suavidade: 0,9

Fig. 1 - Examples of segmentations using different weights for colour and shape.

a) Colour: 1; shape: 0,

b) Colour: 0.2; shape: 0.8; compactness: 0.9; smoothness: 0.1

c) Colour: 0.2; shape: 0.8; compactness: 0.1 ; smoothness: 0.9

b) Os objectos de base superior ou inferior ao nível semântico principal, devem adequar-se nas suas relações de rede, aos objectos superiores ou inferiores que se pretendem classificar. Isto é, existem objectos que apesar de serem classificados numa primeira fase, não têm o grau de desagregação final desejado, mas actuam como base à classificação de objectos deles dependentes, seja em relações de vizinhança ou de hierarquia. Por exemplo, os vários objectos classificados como auto-estrada num nível inferior serão fundidos num só objecto "Auto-estrada" num nível superior.

\section{CLASSIFICAÇÃO DE OBJECTOS: USO DO SOLO URBANO}

A classificação dos objectos, com recurso à segmentação multiresolução permite trabalhar com os conceitos de generalização semântica, procurando-se num nível mais elevado uma precisão das fronteiras dos objectos, e em níveis mais baixos a precisão do detalhe interno dos elementos constituintes dos superobjectos. Falamos por exemplo, na delimitação de uma área de habitação unifamiliar (no nível mais elevado de generalização) e na identificação dos edifícios, jardins, etc., explicativos da mesma (no nível inferior). A segmentação multiresolução permite-nos englobar o conceito de rede hierárquica de objectos, no conjunto de variáveis visuais como a cor, textura, forma, e outros, a utilizar na identificação e descrição dos objectos. Na integração de conceitos, poderemos, então, falar numa associação horizontal e vertical, aquando da delimitação e classificação de polígonos. 
O eCognition trabalha com operadores de lógica fuzzy, o que possibilita o trabalho com a incerteza dos conceitos de linguística patentes na classificação de elementos de espaço, como são disso exemplo os conceitos de "muito" e "pouco", etc. (Benz, et al., 2004). A forma das funções que descrevem o comportamento do objecto num determinado atributo, determina o grau de pertença do objecto a uma classe. O conjunto de operadores disponíveis permite a construção de regras de decisão, com a conjugação de diferentes atributos dos objectos.

Uma classificação inicial dos objectos permite também a correcção da forma dos objectos, recorrendo aos atributos da rede de objectos.

\section{Objectivo e hipóteses}

Como principal objectivo procurou-se diferenciar duas classes de uso do solo, usualmente não separáveis: a habitação unifamiliar e a habitação plurifamiliar. A separação das duas classes deverá ser conseguida recorrendo à parametrização de diferentes dimensões representadas pela natureza do objecto, isto é, a sua resposta espectral, a sua forma, o seu significado e o contexto espacial onde se insere.

A área de estudo (fig. 2), situada no concelho de Oeiras, é uma área periurbana, contígua a Lisboa (capital). As imagens de trabalho são as quatro bandas multiespectrais $^{3}$ do satélite QuickBird (31 Março, 2002), com resolução de 2,88m.

Nesta experimentação foi utilizado o software eCognition (Definiens AG) - object-oriented image analysis. Relativamente à separabilidade das duas classes enunciadas, os pressupostos de partida são:

a. Na imagem as duas classes distinguem-se por dois atributos principais - primeiro a forma e segundo a proximidade espacial com outros objectos ou classes funcionalmente próximas. Por exemplo, é possível encontrar uma proximidade das áreas de jardim pertencentes às áreas de habitação unifamiliar.

b. Na área de estudo predominam os edifícios de telha vermelha e será este o atributo inicial de separação dos edifícios de habitação em relação às restantes classes de ocupação do solo.

Para o tratamento dos dados baseamo-nos nas seguintes hipóteses:

a. Se a habitação unifamiliar apresenta, ao nível do edifício, uma forma próxima do quadrado e se a habitação plurifamiliar apresenta uma forma próxima do rectângulo (por contiguidade espacial dos edifícios); então, parametrizando os dois tipos de formas, é possível obter uma primeira separação das duas classes, ao nível dos edifícios classificados.

3 NIR (Próximo Infravermelho); R (Vermelho); G (Verde); B (Azul). 
b. Se na envolvente aos edifícios classificados como habitação unifamiliar são identificáveis objectos/classes de uso/ocupação do solo como áreas de vegetação (de pequena e média dimensão), passeios, etc., e se a envolvente aos edifícios de habitação plurifamiliar se caracteriza predominantemente por espaço construído de carácter público (parques de estacionamento, por exemplo), então, separando estes elementos é possível obter uma terceira separação das duas classes - agora ao nível dos edifícios e da envolvente, ou seja, das áreas funcionais predominantemente de habitação unifamiliar e de habitação plurifamiliar.

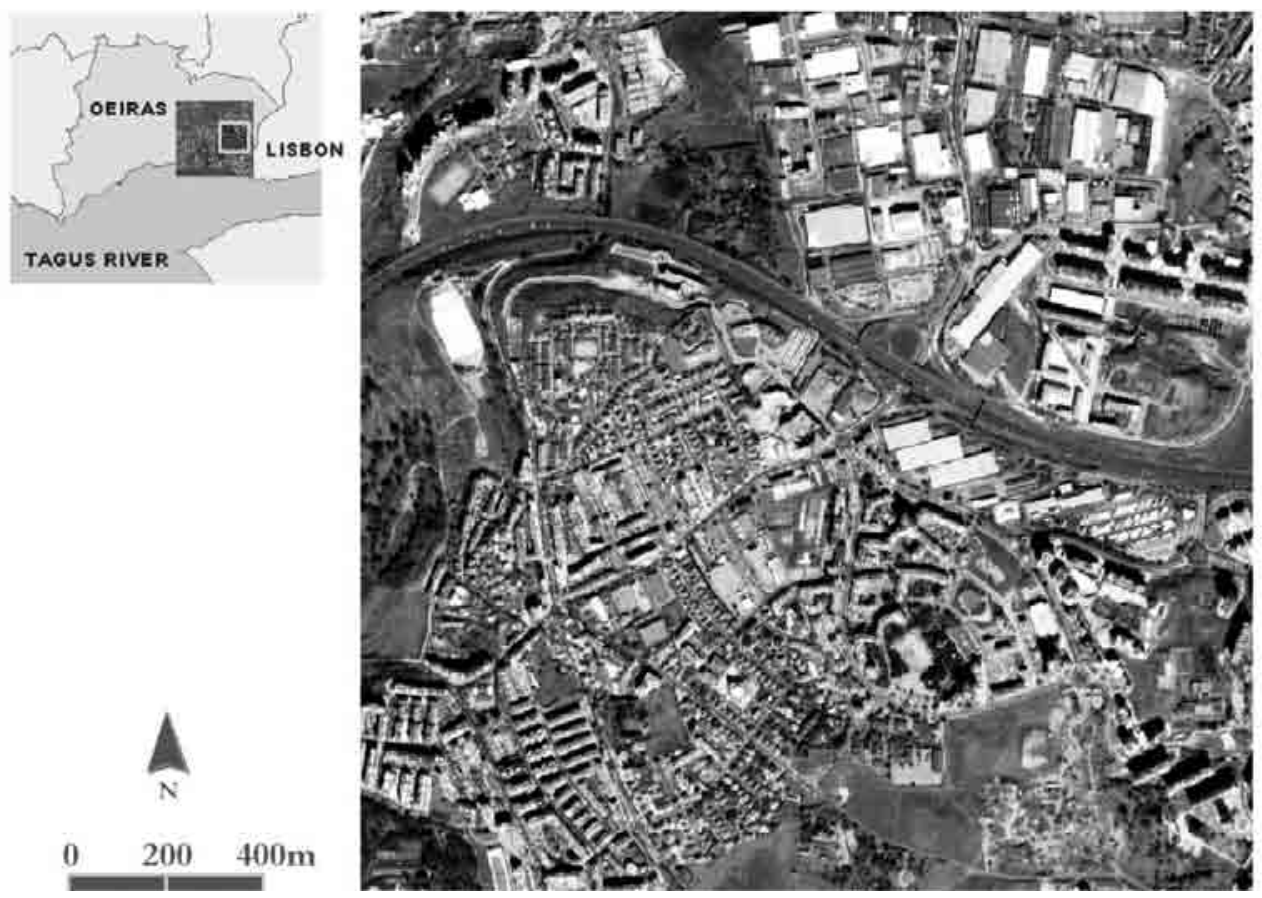

Fig. 2 - Área de estudo: Imagem QuickBird, 31 de Março de 2002 - Linda-a-Velha - Oeiras. Fig. 2 - Study area: QuickBird Image, 31 March 2002 - Linda-a-Velha - Oeiras.

\section{Segmentação da imagem e classificação dos objectos}

Os objectos foram construídos em quatro níveis de segmentação, de forma a conseguir extrair da imagem diferentes estruturas espaço/funcionais, nomeadamente, áreas de diferentes tipologias de habitação, e a separação do construído/não construído. A combinação de parâmetros utilizados para cada um dos níveis é apresentada no Quadro I. 
Quadro I - Parâmetros de Segmentação em 4 níveis

Table I - Parameters for segmentation into 4 Levels

\begin{tabular}{lcccc}
\hline & Nível 1 & Nível 2 & Nível 3 & Nível 4 \\
\hline Parâmetro de escala $^{\mathrm{a}}$ & 10 & 25 & 75 & 100 \\
Cor $^{\mathrm{b}}$ & 0,9 & 0,9 & 0,8 & 0,7 \\
Foma $^{\mathrm{b}}$ & 0,1 & 0,1 & 0,2 & 0,3 \\
Suavidade $^{\mathrm{b}}$ & 0,5 & 0,7 & 0,7 & 0,6 \\
Compactacidade $^{\mathrm{b}}$ & 0,5 & 0,3 & 0,3 & 0,4 \\
\hline
\end{tabular}

a O intervalo de valores é dependente do tamanho da imagem.

b O intervalo de valores varia entre 0 e 1 .

As segmentações finais dos níveis 2 e 3 foram melhoradas com a inclusão das classificações iniciais dos níveis 1 e 4, respectivamente. Tendo sido classificados os edifícios de Telha Vermelha no nível 1, foi possível corrigir, por exemplo, objectos do nível 2 que englobavam telhados e arruamentos num só objecto, através do corte desse objecto, a partir dos limites dos sub-objectos que o constituem. A figura 3 apresenta os limites dos objectos construídos para o nível inferior (nível 1) e para o nível superior (nível 4).
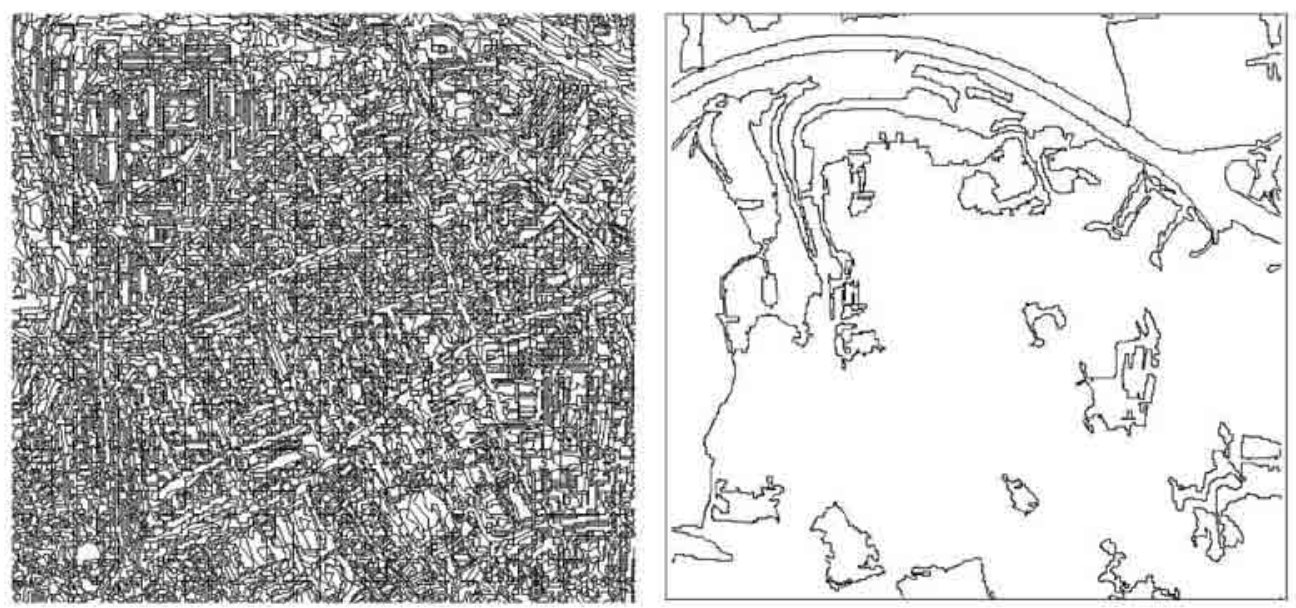

Fig. 3 - Objectos a diferentes escalas de percepção espacial. a) nível 1, b) nível 4. Fig. 3 -Objects at different spatial perception scales. a) Level 1, b) Level 4.

Após a construção dos vários níveis de segmentação, foi iniciada a classificação no nível 1, com a construção de atributos (índices) que separassem os objectos mais significantes na imagem, nomeadamente a Telha Vermelha, a Sombra e a Vegetação (fig. 4). 

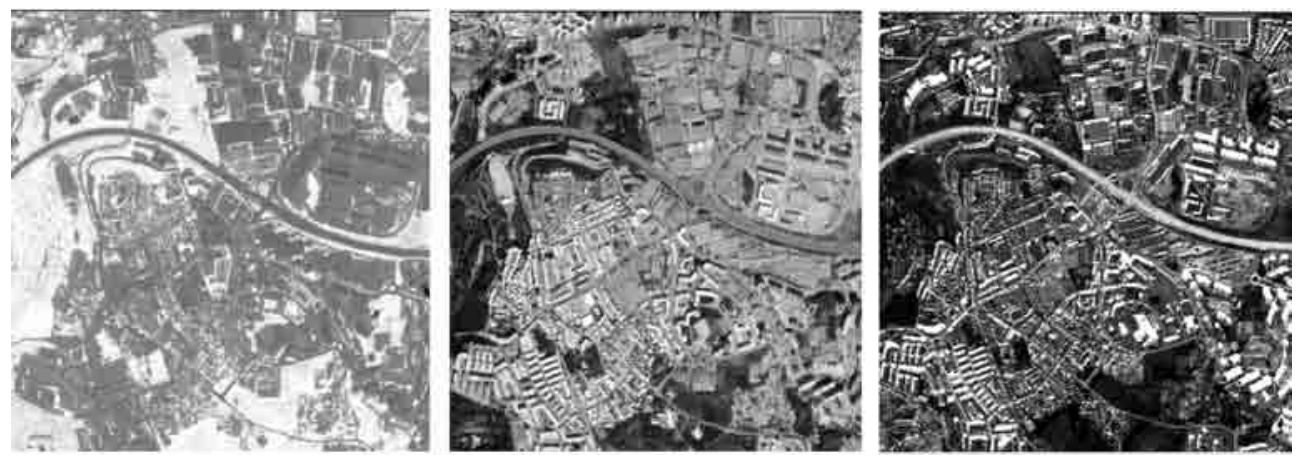

Fig. 4 - Características das classes do nível 1:

a) $\mathrm{NDVI}^{4}$ : [(NIR-R)/(NIR+R)], b) Telha de Barro: [(R-G)/(R+G)],

c) Sombra: $\left[(\mathrm{B}+\mathrm{G}+\mathrm{R}+\mathrm{NIR}) /(\mathrm{NIR})^{2}\right]$.

Fig. 4 - Features of the level 1 classes.

a) NDVI: $[(N I R-R) /(N I R+R)]$, b) Red Tile: $[(R-G) /(R+G)]$,

c) Shadow: $\left[(B+G+R+N I R) /(N I R)^{2}\right]$.

Estes três índices permitiram a classificação das três primeiras classes, respectivamente: Vegetação (a), Telha Vermelha (b) e Sombra (c), necessárias à construção da hierarquia da classificação posterior, na medida em que: i) para destinguir áreas de habitação, procuram-se identificar os edifícios através da telha vermelha; ii) as áreas de habitação unifamiliar apresentam uma continuidade espacial com áreas de vegetação de pequena dimensão (jardins, por exemplo); iii) as áreas de habitação plurifamiliar identificam-se também pela contiguidade espacial da sombra, resultante da altura dos edifícios.

Numa segunda fase foi classificado o nível 4, separando em primeiro lugar o construído do não construído. Assim, foi calculado o NDVI (índice de vegetação da diferença normalizada), para classificar as áreas de vegetação. A classe construído foi determinada com a expressão "todos os objectos não classificados como Vegetação". Numa terceira fase extraiu-se a classe Auto-estrada (como elemento estruturante da organização daquele território), da classe construído. Para a separação desta classe foi utilizado o atributo "Length/Width (line so) (1)" (a qual estabelece a relação entre o comprimento e a largura do objecto, isto é, o seu comportamento linear), no qual são tidos em conta os sub-objectos do nível imediatamente inferior (neste caso o nível 3), para o cálculo do comprimento do objecto. A separação da Auto-estrada no nível 4, permitiu também impedir que os objectos dos níveis inferiores 1 e 2 fossem classificados numa outra classe que não Auto-estrada.

No nível 2 explorou-se o atributo área do objecto, que permite diferenciar os edifícios de habitação unifamiliar dos de habitação plurifamiliar. Para isso, a segmentação do nível 2 foi melhorada para que os limites dos vários objectos do nível 1, classificados como Telha Vermelha e que pertencessem apenas a um 
edifício, fossem agrupados num só objecto no nível 2. Depois desta operação foi construída uma característica que separa os objectos classificados como Telha Vermelha, tendo em conta a dimensão da área que ocupam.

No nível 3, e já numa lógica de classificação de áreas funcionais de uso do solo, foram incluídos os atributos finais de classificação e separação das duas principais tipologias de habitação, ou seja, a relação entre a área relativa de vegetação e a dimensão dos edifícios. Partiu-se do pressuposto de que nas áreas de habitação unifamiliar existe uma maior presença de vegetação em redor do edifício, do que nas áreas de plurifamiliar, onde predominam os espaços construídos públicos, como vias de comunicação e parques de estacionamento.

A classificação final é apresentada na figura 5, tendo especial interesse as duas classes que separam as principais tipologias de uso habitacional.

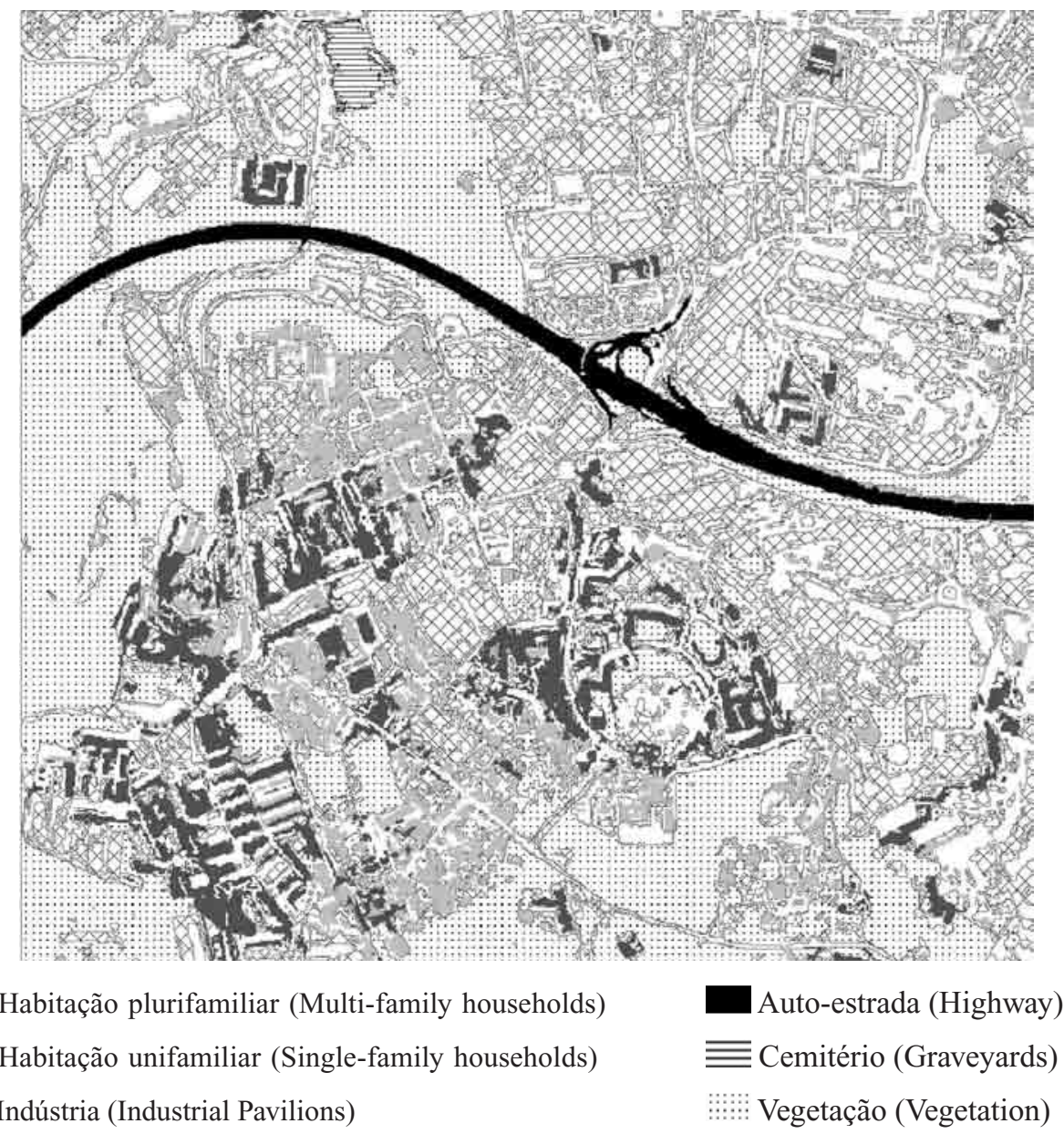

Fig. 5 - Classificação final (nível 3).

Fig. 5 - Final classification (Level 3). 
Numa nota final à classificação desenvolvida salienta-se o facto de que a este nível de abstracção trabalhamos já ao nível conceptual da área e não dos elementos constituintes, pelo que se assume que o detalhe interior não necessita de uma precisão exacta para todos os elementos, mas fundamentalmente para aqueles que permitem estabelecer os limites semânticos e gráficos dos conceitos de área funcional.

De acrescentar que uma posterior sub-classificação da classe sombra, englobando-a nas diferentes classes de uso/ocupação do solo que a mesma afecta, permitirá melhorar, por um lado a delimitação dessas mesmas classes e, por outro lado, ultrapassar um problema de categorização de classes de espaço, já que a classe sombra não é per si nem uso, nem ocupação do solo.

\section{CONSIDERAÇÕES FINAIS}

Após a conclusão da aplicação de uma metodologia exploratória da abordagem orientada por objecto na análise de imagens QuickBird, deparamo-nos com um conjunto de questões de natureza conceptual, mas de implicações operacionais imediatas.

1. Será possível identificar na imagem, objectos por associação com a sua funcionalidade?

2. Será possível identificar na imagem objectos por associação com a sua forma?

3. Será possível identificar objectos, por relações de proximidade e contexto?

Se, por um lado, as primeiras experimentações apontam para respostas afirmativas, às questões anteriores, por outro lado, apontam também para outras questões que requerem futuro desenvolvimento, nomeadamente:

1. Em que medida a escolha dos parâmetros de segmentação determina a construção e significado dos objectos?

2. Qual a influência do tipo de dados para essa mesma construção?

3. Que combinação de parâmetros é adequada para cada classe, ou conjunto de classes, de uso/ocupação do solo?

Assim, face ao trabalho desenvolvido, consideramos que a abordagem orientada por objecto implica uma dupla interpretação, teórica e operacional:

A. Interpretação teórica:

1. O grau de abstracção das entidades de análise é menor, face à simbologia criada na navegação do espaço da imagem, seja ele representativo ou representado, quando comparada com a abordagem pixel a pixel. 
2. Esse mesmo grau de abstracção (escala de percepção espacial) dos objectos é variável dentro de uma mesma imagem, estabelecendo uma relação de implicação com a variação do conteúdo das formas e significados, e consequentemente com as partes que constituem e determinam os objectos compósitos.

3. Existe uma ontologia própria na definição do conceito de objecto, intimamente ligada com a ontologia de imagem, que se antevê de forte desenvolvimento no futuro (por necessidades operacionais), possibilitando alargar o conhecimento das unidades dos objectos, nomeadamente o núcleo e as fronteiras. Veja-se, por exemplo, o trabalho apresentado por Bitter (1999), no qual é discutida a relação entre "rough location, vague defined spatial objects, and indeterminacy of location", partindo das relações entre os objectos e o espaço, e nomeadamente as partes dos objectos e partes do espaço que ocupam.

4. Existe um forte potencial de desenvolvimento em conjugação com as teorias de cognição espacial, inteligência artificial e lógica fuzzy, entre muitas outras. No âmbito da Geografia, a continuação da investigação poderá contribuir para novas formas de entender e trabalhar a modelação de eventos espaciais. Como entender e modelar padrões e estruturas de distribuições espaciais sem um entendimento sistematizado dos objectos criados pela manipulação dos dados ou da informação? Que mutações originamos nos objectos quando integrados em diferentes dimensionalidades, sem que as suas partes sejam totalmente compreendidas? Algumas das questões levantadas por João (1998) sobre as causas e consequências da generalização cartográfica apontam já indícios da dependência entre forma e significado na construção de objectos.

B. Interpretação operacional:

1. Impulsionada sobretudo pelo desenvolvimento e comercialização do software eCognition, existe um rápido crescimento do número de aplicações em diferentes domínios.

2. O software possibilita a construção imediata de objectos com funções atribuídas em relação à sua natureza e aos quais é possível integrar a informação adicional relativamente ao seu papel no território analisado.

3. Embora também dependente da resolução espacial das imagens, assim como da sua resolução espectral, a abordagem orientada por objecto permite o trabalho com diferentes escalas de percepção espacial, com a integração do algoritmo de segmentação multiresolução.

4. Por se tratar de um sistema dinâmico e em aberto, a classificação de imagem pode ser entendida como um processo dinâmico de criação de informação, na medida em que se conjuga a segmentação da imagem com a classificação. 
5. Apoiada na lógica fuzzy permite a integração de valores de incerteza relativamente à discriminação dos objectos geográficos, não só na fase de avaliação de resultados, mas também na fase de classificação. Retomando o tópico da modelação de eventos espaciais, porque não entender o conceito de unidade urbana como um objecto fuzzy, face à sua forma e significado? A certeza da aplicação do conceito a determinado objecto compósito varia em função da maior ou menor proximidade a um núcleo, em relação às suas fronteiras. Desta forma, o processamento da informação pode incorporar valores de incerteza que reflictam a própria subjectividade do conceito, em relação às dinâmicas territoriais encontradas.

\section{BIBLIOGRAFIA}

Baatz M, et al. (2003) eCOGNITION - Object oriented image analysis user guide 3. DEFINIENS IMAGING, München.

Baatz M, Schäpe A (2000) Multiresolution segmentation - an optimization approach for high quality multi-scale image segmentation. In Strobl J, et al. (ed.) Angewandte Geographische Informationsverarbeitung XII. Beiträge zum AGIT-Symposium, Salzburg, Karlsruhe, Herbert Wichmann Verlag: 12-23 [Acedido em 29 de Dezembro de 2003]. http://www.agit.at/papers/2000/baatz_FP_12.pdf

Blaschke T, Strobl J (2001) What's wrong with pixels? Some recent developments interfacing remote sensing and GIS. In GeoBIT/GIS6: 12-17 [Acedido em 29 de Dezembro de 2003]. http://www.definiensimaging.com/documents/publications/GIS200106012.pdf

Câmara G, Egenhofer M, Fonseca F, Monteiro A M V (2001) What's in an image. In Montello D R (ed.) Spatial information theory - a theoretical basis for GIS. COSIT '01, Santa Barbara: 24 [Acedido em 29 de Dezembro de 2003]. http://citeseer.nj.nec.com/camara01whats.html

Haralick R M, Shapiro L G (1985) Image segmentation techniques. In computer vision graphics and image representation, 29: 100-132.

He D C, Wang L, Baulu T, Morin D, Banari A (1994) Classification spectrale et texturale des donnes d'images SPOT en milieu urbain. International Journal of Remote Sensing, 15(10): 2145-2152 .

Hoffman D (2000) Visual intelligence. W. W. Norton \& Company, New York.

Hofmann P (2001) Detecting informal settlements from ikonos image data using methods of object oriented image analysis - an example from Cape Town (South Africa). In Jürgens C (ed.) Remote sensing of urban areas. Fernerkundung in urbanen Räumen. (Regensburger Geographische Schriften, Heft 35). Regensburg: 107-118 [Acedido em 29 de Dezembro de 2003]. http://www.definiens-imaging.com/documents/publications/hofmann.pdf

Jensen-M L (1990) Knowledge-Based classification of an urban area using teture and context information in LANDSAT TM imagery. Phtogrammetric Engineering and Remote Sensing, 56(6): 629-643.

João E M (1998) Causes and consequences of map generalisation. London School of Economics, Taylor \& Francis, London. 
Mark D M, Skupin A, Smith B (2001) Features, objects, and other things: ontological distinctions in the geographic domain. In spatial information theory: foundations of geographic information science (Lecture Notes in Computer Science 2205), D R Montello, Springer-Verlag, Berlin: 488-502 [Acedido em 15 de Janeiro de 2003]. http://ontology.buffalo.edu/smith//articles/COSIT01MSS.pdf

Nunes J (1991) Geographic space as a set of concrete geographical entities. In Mark D M, Andrew U F (ed.) Cognitive and linguistic aspects of geographic space. Kluwer Academic Publishers, Dordrecht: 9-33. 